\title{
COSTOS DE CALIDAD EN EL SECTOR CEMENTERO DEL MUNICIPIO SAN FRANCISCO. CASO: VENEZOLANA DE CEMENTOS
}

\author{
PORTILLO, BILLY \\ THOYA, MAITA \\ STEFANIA, NOYA
}

UNIVERSIDAD RAFAEL URDANETA

VENEZUELA 


\title{
RESUMEN
}

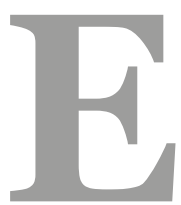

l punto de partida que orienta la presente investigación está delimitado para analizar los costos de calidad del Sector Cementero del Municipio San Francisco. El tipo de investigación es descriptiva, insertada dentro del diseño de campo no experimental, y transversal, tomando como población la empresa Venezolana de Cementos. Se aplicó un cuestionario a través del cual se evidenció una orientación inadecuada para la identificación de las actividades que generan los costos de calidad. Pudiendo concluir que no existe el buen manejo de los mismos; teniendo el abordaje del tema de costos de calidad en la empresa estudiada muchos vacíos.

Palabras Clave: Costos de Calidad, Cemento, Fallas internas, Fallas externas.

\section{COST OF QUALITY IN THE MUNICIPALITY OF SAN FRANCISCO CEMENT}

\begin{abstract}
The starting point that guides this research is delimited to analyze quality costs Township Cement Sector San Francisco. The research is descriptive, inserted into the non-experimental field design, and cross, on the Venezuelan population Cement Company. A questionnaire through which is evidenced inadequate guidance to identify the activities that generate quality costs. We can conclude that there is no proper management thereof; taking addressing the issue of quality costs in the company studied many gaps.
\end{abstract}

Keywords: Quality costs, cement, internal failures, external failures.

\section{INTRODUCCIÓN}

Una de las herramientas que las organizaciones han utilizado para mantener el control sobre su efectividad, son los costos de calidad, así lo expresa Ibarra (1997) cuando sostiene que, las organizaciones se esfuerzan por mantener la efectividad con el fin de conservar una posición favorable en el mercado, lograda a través de la calidad de los productos y servicios, así como la satisfacción de los clientes, aunado al control de los costos generados en este empeño.

En tal sentido, los costos de calidad según Dale (1993), constituyen una herramienta gerencial, de carácter contable, que permite a la alta dirección detectar los datos necesarios para identificar y clasificar los gastos en los cuales incurre la organización, conociendo así la mag- 
nitud del problema de los costos; medir e identificar con precisión en términos económicos las áreas de oportunidad; evaluar en valores los resultados de los esfuerzos de las medidas tomadas acorde con el programa de mejora continua de la calidad que está implementando la organización, optimizar los esfuerzos por lograr mejores niveles de calidad, costos y/o servicios que incrementen su competitividad y afirmen su permanencia en el mercado.

Por esta razón, al hablar de calidad de un producto, es necesario que se definan los costos que traen consigo elaborar y mantener un producto que permita aumentar la competitividad y sustentabilidad de la organización. Usualmente se considera que el costo de calidad lo integran las actividades inherentes con el aseguramiento, a la detección de errores y desechos en la línea de producción; no obstante, el concepto ha evolucionado y se entienden como costos de calidad aquellos incurridos en prevención de fallas, implementación, operación, mantenimiento y evaluación de los sistemas de calidad, incluyendo los referentes a sistemas de mejoramiento continuo. Específicamente este tipo de costo trata sobre costos de prevención, de fallas internas o externas así como de evaluación (Harrington, 2003).

Es necesario acotar que gran porcentaje de los costos de calidad se desembolsan para cuantificar la calidad, así como para estimar el costo de las fallas. Estos gastos se suman al valor de los productos o servicios que paga el consumidor y aunque este último solo los percibe en el precio, llegan a ser importantes para él, cuando a partir de la información que se obtiene, se corrigen las fallas y se disminuyen los incumplimientos, reprocesos, y a consecuencia de estos ahorros se disminuyen los precios (Colunga y Saldierna, 1994).

Ahora bien, según los autores ya citados anteriormente, cuando no hay quien se preocupe por los costos, simplemente se repercuten al que sigue en la cadena (proveedor, productor, distribuidor, intermediario y consumidor), hasta que surge un competidor que ofrece costos inferiores. En lo que no siempre se recapacita, es en que, el costo de devoluciones, que implica el regresar o destruir mercancía, el papeleo y su reposición al comerciante, lo pagan finalmente todos los clientes.

Por lo mencionado anteriormente, el presente artículo pretende describir los costos de calidad en la empresa del sector cementero del municipio San Francisco del Estado Zulia, específicamente en la empresa Venezolana de Cementos como caso de estudio, con la finalidad de conocer si la industria cementera utiliza los costos de calidad como una fuente que representa la mejora en la competitividad y la reducción de erogaciones en las que incurre constantemente.

El tipo de investigación es descriptiva, pues busca especificar los tipos de costos de calidad que son aplicados en el sector cementero evaluando el costo total de la calidad integrado por los costos de prevención, de evaluación, de fallas internas y fallas externas. En cuanto al diseño de la investigación, este se inserta en el de campo, pues, la información fue obtenida directamente de la realidad de la empresa cementera, en cuanto al diseño es no experimental transeccional ya que, no se tiene control alguno sobre la variable en cuestión. La población tomada estuvo representada por la empresa Venezolana de Cementos ubicada en el Municipio 
San Francisco del Estado Zulia. Se aplicó un cuestionario conformado por 50 ítems que fueron respondidos por el Contador del departamento de costos y el Gerente de calidad mediante una escala de respuesta tipo Likert.

\section{COSTOS DE CALIDAD Y SU TIPOLOGÍA: ABORDAJE TEÓRICO}

Antes de comenzar a explicar claramente definiciones referentes a los costos de calidad es importante que se tenga conocimiento sobre el significado de dos puntos clave como lo son los costos y la calidad. Según Hansen y Mowen (2004), el costo es el efectivo o equivalente de efectivo que se sacrifica para obtener bienes y servicios que se espera que aporten un beneficio actual o futuro para la organización; por otro lado, los mismos autores, ya citados indican que la calidad de un producto o servicio significa que esté de acuerdo con un estándar previamente especificado.

Ahora bien, aclarado el significado de ambos conceptos tanto costos como calidad, es momento de desarrollar y profundizar sobre los costos de calidad. Las ideas acerca del costo de calidad han venido evolucionando rápidamente en los últimos años. Anteriormente era percibido como el costo de poner en marcha el departamento de aseguramiento de la calidad, la detección de costos de desecho y costos justificables (Dale y Plunkett, 1992, citado en Jimenez, 2000).

Existen varias tipos de costos como aquellos ineludibles debido a que son propios de los procesos productivos, los costos indirectos, entre muchos otros; sin embargo, el autor Juran (2001), hace mención a otra tipología, como los denominados costos de calidad, los cuales define como aquellos derivados de los esfuerzos de la organización para fabricar un producto o generar un servicio con la calidad ofrecida.

En conclusión, para efectos de la presente investigación se entiende como costos de calidad, aquellos referentes a todos los esfuerzos que realiza una organización para fabricar un producto o servicio que cuente con los estándares establecidos de calidad, estos varían de acuerdo al tipo de organización o industria, mencionando donde es más provechosa una acción correctiva, permitiendo al mismo tiempo identificar los procesos operativos junto con su eficacia.

Llama poderosamente la atención en realizar un estudio acerca de los tipos de costos de calidad, en la cual existe concordancia entre autores a objeto de la investigación, por consiguientes es tomada como perspectivas más acorde y completa las de los autores Colunga y Saldierna (1994) y Amat (2005) quienes los dividen bajo dos clasificaciones, siendo la primera de ellas los costos de calidad y la segunda referente a los costos de no calidad.

En cuanto a la primera clasificación, los autores ya citados, mencionan que los costos de calidad cuentan con una subdivisión, la misma es la siguiente: a) costos de prevención, siendo aquellos importes erogados para prevenir y evitar el incumplimiento de los requerimientos en cualquier producto, servicio, proceso y/o sistema de la empresa. La mejor forma de invertir el dinero en una empresa es canalizando las inversiones hacia los costos por prevención, ya que su uso adecuado contribuye a la disminución de las otras clases de costos; b) los costos de evaluación, son las erogaciones generadas por la inspección de los productos, los servicios, los 
procesos y/o los sistemas para asegurar que cumplen con los requerimientos. Estos costos se implementan siempre que la alta dirección no está segura de que la inversión para la prevención es capaz de eliminar al 100\% la posibilidad de error.

Por otro parte, en cuanto a la segunda clasificación los autores indican que los costos de no calidad son aquellos ocasionados por no cumplir con los requerimientos de los productos, servicios, procesos y/o sistemas. Cuentan con una subdivisión la cual está representada por: a) costos de fallas internas, siendo aquellos importes generados por no cumplir con los requerimientos de los productos, servicios, procesos y/o sistemas en donde la organización tiene un control directo; y b) costos por fallas externas, aquellos erogados por no cumplir con los requerimientos de los productos, servicios, procesos y/o sistemas no controlados directamente por la empresa.

Las fallas pueden reducirse a un mínimo si se vigilan cuidadosamente las dimensiones "estándares" en las materias primas y utilizando técnicas como los ajustes asistidos por computadoras, para aprovechar en su totalidad los materiales existentes. Se ilustra en el cuadro $\mathrm{N}^{\mathrm{o}} 1 \mathrm{y}$ cuadro $\mathrm{N}^{\mathrm{O}} 2$ actividades que conforman los costos de calidad y no calidad respectivamente.

Cuadro $\mathrm{N}^{\circ} 1$ Actividades que conforman los Costos de Calidad

\begin{tabular}{|l|l|}
\hline $\begin{array}{l}\text { TIPOS DE COSTOS } \\
\text { DE CALIDAD }\end{array}$ & \multicolumn{1}{c|}{ MODELOS } \\
\hline \multirow{2}{*}{ PREVENCIÓN } & $\begin{array}{l}\text { - Costos de Planeación. } \\
\text { - Costos de Capacitación y formación. } \\
\text { - Costos de revisión de procesos, vigilancia y control de equipos. } \\
\text { - Costos necesarios para monitorear los niveles de mejoramiento alcanzados. } \\
\text { - Evaluación y asesoría de proveedores. }\end{array}$ \\
\hline \multirow{2}{*}{ EVALUACIÓN } & $\begin{array}{l}\text { - Inspección de entrada de insumos. } \\
\text { - Inspección al proceso de producción. } \\
\text { - Inspección del producto final. } \\
\text { - Evaluación de impacto y calificación del servicio. }\end{array}$ \\
\hline
\end{tabular}

Fuente: Gómez (2005).

Cuadro № 2 Actividades que conforman los Costos de No Calidad

\begin{tabular}{|l|l|}
\hline $\begin{array}{l}\text { TIPOS DE COSTOS } \\
\text { NO DE CALIDAD }\end{array}$ & \multicolumn{1}{c|}{ MODELOS } \\
\hline \multirow{2}{*}{ FALLAS INTERNAS } & $\begin{array}{l}\text { - Desperdicios causados por fallas en el proceso. } \\
\text { - Reproceso para corregir los productos defectuosos. } \\
\text { - Costos por desechos, por no poder repetirse el proceso. } \\
\text { - Costos por tiempo ocioso, maquinaria por defectos de mantenimiento o de } \\
\text { materia prima. }\end{array}$ \\
\hline FALLAS EXTERNAS & $\begin{array}{l}\text { - Costos asociados a devolución de productos y reclamaciones de los clientes. } \\
\text { - Costos de garantía, reparaciones y menor grado de confiabilidad y prestigio. }\end{array}$ \\
\hline
\end{tabular}

Fuente: Gómez (2005). 


\section{COSTOS DE CALIDAD EN LA EMPRESA CEMENTERA. CASO DE ESTUDIO VENEZOLANA DE CEMENTOS}

A continuación se muestra los resultados obtenidos para cada uno de los Costos de Calidad: Costos de Prevención, Costos de Evaluación, Costos de Fallas Internas, Costos de Fallas Externas.

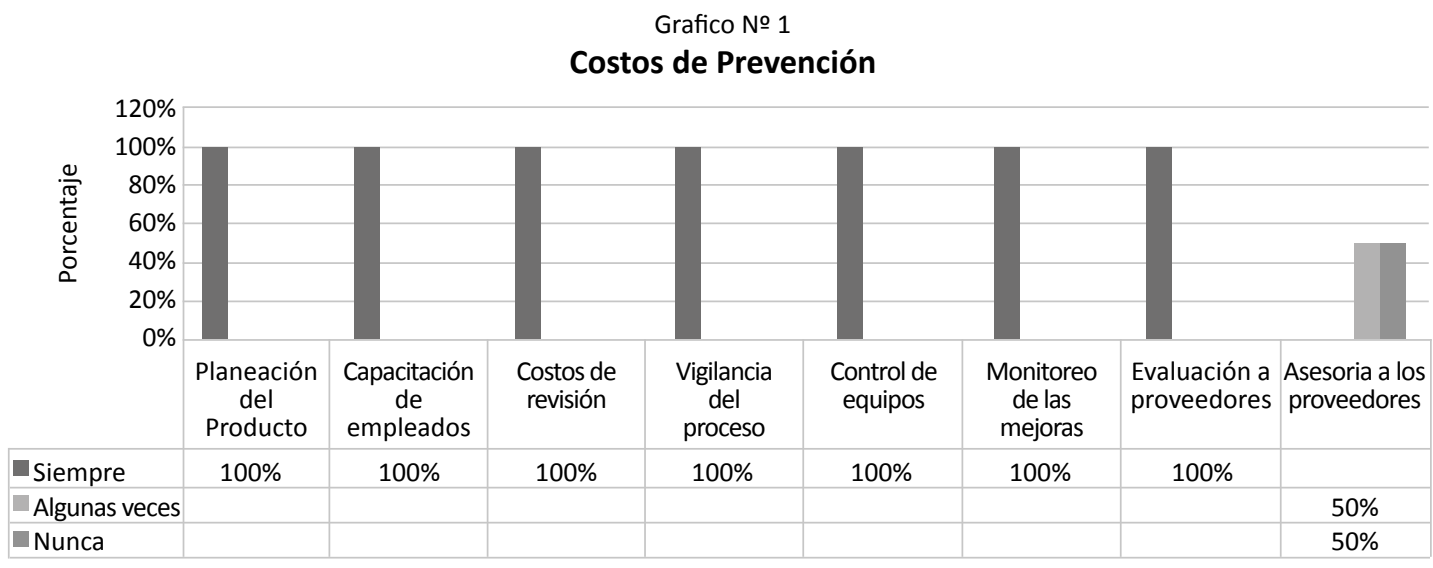

Como se muestra en el grafico $\mathrm{N}^{0} 1$ hubo concordancia en las respuestas arrojadas por los encuestados señalando que siempre se realizan actividades relacionados con la planeación en la elaboración del producto, así como programas de capacitación o formación para los empleados, se incurren en costos causados por revisión del proceso productivo llevando una vigilancia del mismo para su correcta elaboración. Del mismo modo siempre se lleva un control sobre los equipos utilizados en la producción, monitoreando los niveles de mejoramiento en el proceso productivo, realizando evaluaciones a los proveedores.

Mientras que hubo discrepancia donde uno de los encuestados señala que algunas veces se realizan asesorías a los proveedores y el otro porcentaje restante indica que nunca se realiza esta actividad. Para Gómez (2005), todas las actividades señaladas son consideradas como costos de prevención los cuales define Amat (2005), Colunga y Saldierna (1994), como aquellos conformados por acciones llevadas a cabo para evitar defectos en el diseño y desarrollo del producto. En el caso de Venezolana de Cementos, llama la tención que en cuanto al recurso humano, aún cuando éste se encuentra calificado y en constante capacitación y adiestramiento, no contribuye para el reconocimiento y posterior clasificación de costos de calidad. 


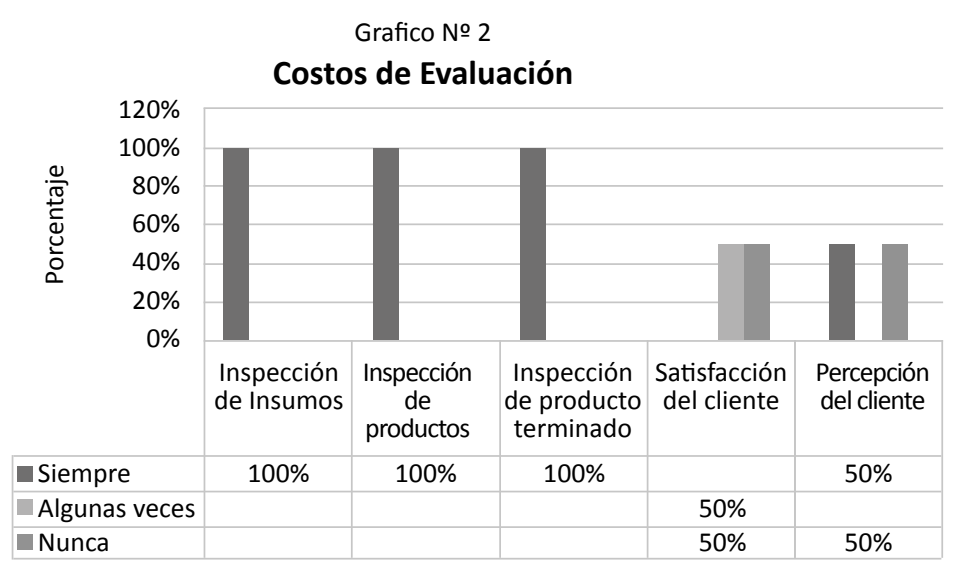

En el grafico $\mathrm{N}^{\circ} 2$ se muestra que los encuestados estuvieron de acuerdo al indicar que siempre la empresa realiza inspecciones a la entrada de insumos, al proceso de producción y al producto final, esto se encuentra representado por el 100\%. En el caso de la medición de la satisfacción del cliente al obtener el producto final el 50\% señala que casi siempre es llevado a cabo, mientras que el otro porcentaje respondió algunas veces.

Para el ítem que hace referencia a la percepción del cliente en cuanto a la calidad del producto final, las opciones de repuestas estuvieron divididas en 50\% para la alternativa siempre y 50\% para la alternativa algunas veces. Para Gómez (2005) todas las actividades señaladas conforman a los costos de evaluación, definidos por Amat (2005), Colunga y Saldierna (1994), como aquellas erogaciones generadas por la inspección de los productos, servicios, procesos, y/o los sistemas para asegurar que se cumplan con los requerimientos. Es importante reseñar que existe un débil seguimiento a las políticas de satisfacción del cliente en la empresa Venezolana de Cementos, posiblemente ocasionado por la falta de competencia en la región donde desarrolla sus actividades.

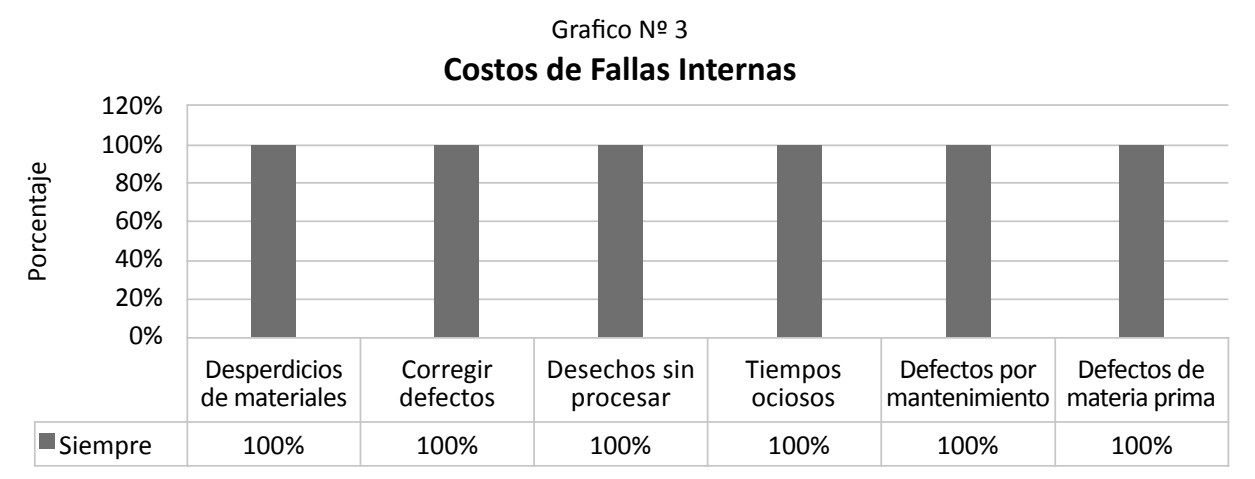

Tal como se puede observar en el grafico $\mathrm{N}^{\circ} 3$ todas las respuestas de los encuestados coinciden al señalar que siempre la empresa considera los desperdicios causados por fallas en el proceso productivo, así como los costos generados por tiempo ocioso, por reprocesos para corregir los productos defectuosos; del mismo modo considera los costos de los desechos por 
no repetir el reproceso, costos que representan las máquinas por defectos de mantenimiento y lo defectos en la materia prima; estando todo esto representado por el 100\%.

De acuerdo con Gómez (2005) todas las actividades señaladas conforman a los costos de fallas internas, definidos por Amat (2005), Colunga y Saldierna (1994), como aquellos importes generados por no cumplir con los requerimientos del producto, servicios, procesos y/o sistemas en donde la organización tiene un control directo. En dicha industria, existen los controles apropiados para el aseguramiento de los estándares requeridos en la fabricación de cemento, lo que contribuye a facilitar el trabajo para la identificación de los costos de calidad.

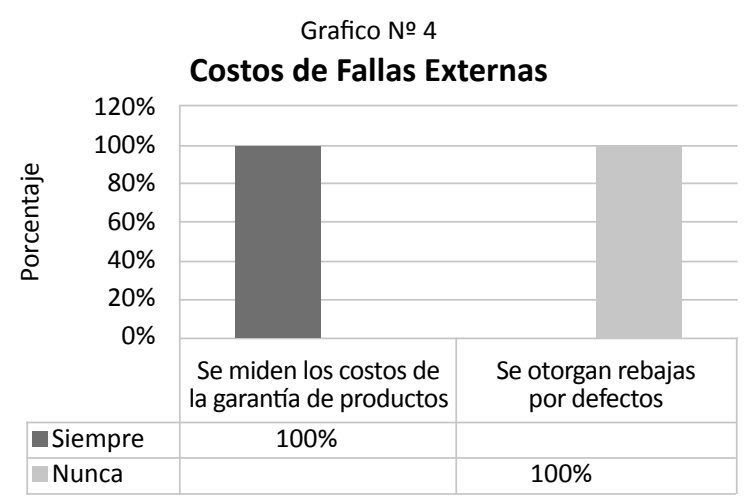

El 100\% de los encuestados tal como se muestra en el grafico $\mathrm{N}^{\mathrm{o}} 4$ estuvo de acuerdo al indicar que siempre se consideran los costos asociados a las garantías del producto, de igual forma se estuvo de acuerdo al señalar que nunca se considera otorgar algún tipo de rebaja por un defecto que pueda presentar el producto ya que estos se aseguran que la entrada de materiales, el procesamiento de los mismos y el producto final como tal sean inspeccionados y controlados adecuadamente.

De acuerdo con Gómez (2005), todas las actividades señaladas conforman a los costos de fallas externas, definidos por Amat (2005), Colunga y Saldierna (1994), como importes generados por no cumplir con los requerimientos del producto, servicios, procesos y/o sistemas no controlados directamente por la empresa.

En Venezolana de Cementos, no se consideran rebajas para los clientes, en virtud de que posiblemente ante la ausencia de competencia en el mercado, ha debilitado las políticas dirigidas al cliente, así como el establecimiento de un control de precios por parte del Estado Venezolano.

\section{A MANERA DE CONCLUSIÓN}

Una vez analizado los resultados se puede concluir que, no existe en la organización los conocimientos sólidos y las disposiciones que se necesitan para llevar un manejo adecuado en referencia a los costos de calidad. La empresa a pesar de conocer cuantitativamente el valor de los costos de calidad total, no cuenta con las herramientas necesarias para identificar qué 
actividad, acción, error, o reproceso pertenece a cada uno de los costos de calidad, sabiendo que éstos son los costos de prevención, costos de evaluación, costos de fallas internas y externas. En este caso es oportuno que la empresa aproveche el conocimiento de la clasificación de costos de calidad para incorporar en su sistema de contabilidad, los respectivos códigos en su plan de cuentas.

Si bien la industria Venezolana de Cementos realiza un sin fin de actividades a las que el sector cementero no escapa, como se pudo evidenciar, se llevan a cabo actividades relacionadas con la planeación en la elaboración del producto, programas de capacitación o formación para los empleados, considerando los costos causados por revisión del proceso productivo, así como la vigilancia del proceso productivo para su correcta ejecución, control sobre los equipos utilizados, se mantiene un monitoreo de los niveles de mejoramiento en el proceso productivo, se realizan evaluaciones a los proveedores así como también inspecciones a la entrada de insumos al proceso de producción, e inspecciones al producto final. Es por ello que se hace necesaria la documentación y seguimiento del control de las mismas, en virtud de que son actividades que le agregan valor al producto y que a la larga contribuyen a reforzar el proceso de mejoramiento continuo.

Se consideran los desperdicios causados por fallas en el proceso productivo, de igual forma los reprocesos para corregir los productos defectuosos, los costos de los desechos por no repetir el reproceso son tomados en cuenta, así como los costos generados por tiempo ocioso, aquellos costos que representan las máquinas por defectos de mantenimiento, los defectos en la materia prima, los costos asociados a las garantías del producto considerando otorgar alguna rebaja al cliente en caso que éste tenga algún defecto; acciones y actividades todas propias de cada uno de los diferentes costos de calidad. Todos costos de fallas internas, que se generan por no hacer las cosas bien. Si la empresa Venezolana de Cementos identifica claramente la importancia de controlar este tipo de costos, tendrá como resultado las estrategias que debe aplicar a fin de mejorar sus procesos.

Por consiguiente, se puede decir que están presentes los costos de calidad en la empresa Venezolana de Cementos, solo que ésta no considera utilizar la clasificación de las actividades que conforman cada uno de ellas. Por lo anteriormente dicho, es importante que la empresa tome correcciones, ya que, una adecuada identificación del costo de la calidad, unido a las consiguientes acciones de mejora, trae como resultado un menor costo, mayor trabajo de valor añadido para el cliente y recursos disponibles para otras actividades. El costo de la calidad no es exclusivamente una medida absoluta del desempeño, su importancia estriba en que indica donde será más redituable una acción correctiva para una empresa.

El trabajo con estos costos permite centrar la atención en asuntos en los que se gastan grandes cantidades, y detectar las oportunidades que en potencia pueden ayudar a reducir gastos, facilita medir el desempeño y constituye una base para la comparación interna entre productos, servicios, procesos, departamentos y externa con la competencia; además ayuda a los directivos a justificar cualquier posible mejoramiento de la calidad esto según el autor Dale (1993). 
Al trabajar de forma adecuada con los costos de calidad, se tendrá una estrategia para lograr la eficacia en todas las actividades dentro de la organización, se podrá identificar plenamente dónde se encuentran los escollos y las zonas de problemas, a la vez que se podría establecer los controles y medios para subsanar dichos problemas, y de ésta manera conocer el costo de obtener la calidad y cuanto representaría no tenerla; de ahí que, el conocimiento de los costos de calidad es esencial para lograr la eficiencia y la eficacia en la compañía.

Para Venezolana de Cementos, el manejo adecuado y oportuno de la información relacionada con los costos de calidad debidamente clasificados, es una oportunidad para establecer acciones, que permitan contribuir con el aprovechamiento de recursos de manera apropiada, por lo cual se hace notoria la importancia que tiene el aprovechar al máximo las bondades que ofrece el uso de la información que genera el análisis de costos de calidad, los cuales constituyen una herramienta que permite a largo plazo generar ventajas competitivas sostenibles; entre éstas la misma reducción de costos de calidad puede convertirse en un factor clave para obtener y mantenerse en los mercados.

\section{REFERENCIAS BIBLIOGRAFICAS}

Amat, Oriol (2005). Costos de Calidad. Costes de Calidad y No Calidad. Ediciones 2000 S.A.

Colunga, Carlos y Saldierna, Arturo (1994). Los Costos de Calidad. México; DF Editorial Panorama.

Dale, Barrie G (1993). Costos de calidad. México D. F. Grupo Editorial Iberoamericana.

Gómez, O. (2005). Contabilidad de Costo. Costo de Calidad. Quinta Edición. Bogotá; Colombia. Editorial McGraw-Hill.

Hansen, Don R y Mowen, Maryanne M (2004). Administración de costos: Contabilidad y control. México; D.F. Editorial Internaciónal Thomson Editores.

Harrington, H. (2003). El Costo de la Mala Calidad. México; DF. Ediciones Díaz Santos.

Ibarra, Gallardo (1997). La empresa ante la Hiperturbulencia. Mexico; D.F. Innovación, Año 9, $\mathrm{N}^{\circ} 1$.

Juran, Joseph (2001). Manual de Calidad. Quinta Edición. Barcelona; España. Editorial McGraw-Hill. 
Mallo, C. y Jimenez, M.A (200o). Contabilidad de Costes, Madrid; España, Editorial Pirámide.

\section{CURRICULUM VITAE Billy Portillo}

Licenciado en Contaduría Pública. Docente Universitario en la Cátedra de Contabilidad de Costos. Universidad Rafael Urdaneta, Maracaibo, Venezuela.

billyportillo@gmail.com

\section{Thoya Maita}

Licenciada en Contaduría Pública. Contador Público de libre ejercicio. Venezuela. thoyamaita@hotmail.com

\section{Stefania Noya}

Licenciada en Contaduría Pública. Contador Público de libre ejercicio. Venezuela. stefanianoya@hotmail.com 